\title{
Deutschsprachige Lexikographie in den böhmischen Ländern und der Sprachpurismus Sommers und Demuths Wörterbücher vor dem Hintergrund der Verdeutschungspraxis'
}

\author{
Eva JANEČKOVÁ
}

\begin{abstract}
German lexicography in the Bohemian Lands and language purism: Sommer's and Demuth's dictionaries in the context of Germanisation processes

This study focuses on two German dictionaries published in the Bohemian Lands during the 19th century, whose titles show their relatedness to language purism. In the first part of the study, both dictionaries are presented and compared based on various criteria such as their authors' motivation for publishing them and the presence of any aspects of Germanisation processes. The second part contains an analysis of two parts of the dictionaries; the aim is to examine the dictionary entries and identify the authors' personal stance on language purism.
\end{abstract}

Keywords: linguistic purism, Germanisation, lexicography

DOI: doi.org/10.15452/StudiaGermanistica.2021.28.0001

\section{Einleitung}

Das 19. Jahrhundert zeichnete sich auf dem Gebiet des heutigen Deutschlands durch ein steigendes Interesse für die eigene Muttersprache aus. Bereits am Anfang des Jahrhunderts wurde das Verdeutschungswörterbuch von Joachim Heinrich Campe herausgegeben, das zu den einflussreichsten Werken dieser Art zählt und weitere Autoren zur Verfassung ähnlicher Werke ermutigte (vgl. Polenz 1994:126-133, Schiewe 1988). Die sprachpuristische ${ }^{2}$ Tätigkeit blieb jedoch noch lange Zeit auf die Arbeit von einzelnen Gelehrten beschränkt. Das änderte sich nach der Reichsgründung, seitdem diese Bestrebungen neuerdings vom Staat unterstützt wurden. Es stieg die Anzahl puristischer

Der Beitrag entstand dank der finanziellen Unterstützung des MŠMT ČR, die der Palacký-Universität Olomouc gewährt wurde (IGA_FF_2020_029).

2 In dieser Studie wird der Terminus „Fremdwortpurismus“, der sich oft auf eine radikale Verdeutschungsarbeit bezieht, vermieden und die mildere Bezeichnung „Sprachpurismus“ bevorzugt (vgl. Kirkness 1998: 407, Polenz 1994: 107 f.). 
Arbeiten, deren Ziel in der Vermeidung nichtindigener Ausdrücke in der deutschen Sprache bestand und deren Titel die Termini „Verdeutschung“ oder „,verdeutschend“ enthielten. Mit der Herausgabe solcher Arbeiten befasste sich nach seiner Gründung im Jahre 1885 insbesondere der Allgemeine Deutsche Sprachverein, der einen institutionellen Rahmen bot und organisierte Beschäftigung mit der Verdeutschungsarbeit ermöglichte. ${ }^{3}$ Des Weiteren gelang es dem Verein, zahlreiche Zweigvereine außerhalb des zentralen deutschen Sprachraums zu gründen, und dadurch deutsche Bevölkerung aus diesen Gebieten für die Verdeutschungsarbeit zu gewinnen. Das betraf auch die böhmischen Länder, wo der Höhepunkt der puristischen Bestrebungen zweifellos mit der Tätigkeit des Allgemeinen Deutschen Sprachvereins zusammenhing. ${ }^{4}$

Dass es in den böhmischen Ländern auch vor dem Allgemeinen Deutschen Sprachverein $\mathrm{Pu}-$ risten gab, die sich für die Reinheit der deutschen Sprache einsetzten, wird in dieser Studie gezeigt: Zwei vergessene Wörterbücher, in deren Titeln die Wörter „Verdeutschung“ bzw. ,,verdeutschend“ vorkommen, werden einem Vergleich unterzogen. Es wird untersucht, inwieweit sich die Einstellung der Autoren zur Verdeutschungsarbeit in der Gestaltung ihrer Werke widerspiegelt und ob die Nachschlagewerke mit den Termini „Verdeutschung“ und „,verdeutschend“ im Titel Auskunft über die puristischen Motive der Autoren geben.

Die Studie besteht aus einem theoretischen und einem praktischen Teil. Im ersten Teil wird der Einfluss der puristischen Tendenzen auf den Metateil des Wörterbuchs bzw. die Struktur der Artikel untersucht und nach den Verdeutschungsprogrammen und Motiven der Verdeutschungsarbeit gefragt. Der praktische Teil befasst sich mit der Analyse der Lemmata, die sich in den Wortstrecken E und $\mathrm{L}$ befinden.

\section{Analysierte Wörterbücher}

Bevor auf die Analyse eingegangen wird, müssen zuerst die behandelten Wörterbücher sowie ihre Autoren kurz vorgestellt und in den Kontext der puristischen Bestrebungen in der damaligen Zeit eingeordnet werden. Außerdem wird auf eine mögliche Klassifizierung von fremdwortbezogenen Wörterbüchern hingewiesen.

Das erste behandelte Wörterbuch trägt den Titel ,Neuestes wort- und sacherklärendes Verdeutschungswörterbuch aller jener aus fremden Sprachen entlehnten Wörter, Ausdrücke und Redensarten, welche die Teutschen bis jetzt, in Schriften und Büchern sowohl als in der Umgangssprache, noch immer für unentbehrlich und unersetzlich gehalten haben; verbunden mit einer Erklärung auch der weniger bekannten Kunstwörter und andern Ausdrücke der teutschen Sprache. Ein höchstnützliches Handbuch für Geschäftsmänner, Zeitungsleser und alle gebildete Menschen [!] überhaupt‘. Das Wörterbuch, das Johann Gottfried Sommer schrieb, wurde in Prag veröffentlicht. In diese Analyse wurde die zweite Auflage des Wörterbuchs aus dem Jahre 1819 einbezogen (vgl. Janečková 2020). Das zweite Wörterbuch, ,Vollständigstes verdeutschendes und erklärendes Fremdwörter-Buch der deutschen Schrift= und Umgangssprache, mit Bezeichnung der richtigen Aussprache, Betonung, Abstammung, Rechtschreibung und Bildung der Wörter, so wie erklärendes Wörterbuch aller in der deutschen Sprache gebräuchlichen Wörter der Asthetik [!], Musik, Technik, Schifffahrtskunde, Botanik, Naturwissenschaft, Kriegswissenschaft, Eigennamen etc.', wurde von Joseph Demuth verfasst und von H. F. Pardatscher im Jahre 1853 im Verlag von Carl Winiker in Brünn veröffentlicht. Auch in diesem Fall handelte es sich nicht um die erste Auflage. ${ }^{5}$

Der Verein widmete sich der Pflege der deutschen Sprache, die sich nicht nur auf Verdeutschungsarbeit beschränkte. Er wurde von Hermann Riegel gegründet (vgl. Hauffen 1916/1917).

$4 \quad$ Es gab z. B. auch Zweige in Russland, England, Italien oder Belgien (vgl. Hauffen 1916/1917).

5 Die Werbung für das Wörterbuch wurde bereits im Jahre 1850 in der Zeitung ,Bote für Tirol und Vorarlberg' publiziert und es gab auch eine Anmerkung in den Börsenblättern im Jahre 1852 (URL 1, URL 2). Sommers Wörterbuch wurde noch in den Jahren 1825, 1833 und 1839 herausgegeben (vgl. Janečková 2020). In den Börsenblättern gab es eine Werbung für Demuths Werk noch im Jahre 1855 (eig. eine Ausgabe aus dem Jahre 1854) und Carl Winiker warb für dieses Wörterbuch noch im Jahre 1872 (URL 3, ULR 4). 
Bereits der flüchtige Vergleich beider Werke liefert erste Unterschiede. Der Unterschied zwischen den Wörterbüchern besteht in ihrem Umfang und in der Struktur der Wörterbuchartikel. Sommers Wörterbuch beträgt etwa 551 Seiten. Im Metateil des Wörterbuchs befinden sich der, Vorbericht zur ersten Auflage', die ,Vorrede zur zweiten Auflage', das Abkürzungsverzeichnis und eine Liste mit Druckfehlern und Verbesserungen. Die Lemmata im Wörterbuchteil sind in zwei Spalten aufgeteilt und es überwiegt die alphabetische Anordnung. Dagegen erstreckt sich Demuths Werk über 876 Seiten und die Lemmata sind in drei Spalten aufgeteilt. Die Anzahl der Lemmata übertrifft also Sommers Auswahl um ein Vielfaches (s. weiter). Im Metateil des Wörterbuchs kommen lediglich eine kurze Vorrede und das Abkürzungsverzeichnis vor. Die Lemmata sind auch hier überwiegend alphabetisch angeordnet.

Sommer gestaltete seine Wörterbuchartikel sehr einfach. ${ }^{6}$ Sie enthalten Äquivalente und Paraphrasen als die obligatorischen Bestandteile. Weniger häufig erscheinen dann die Angabe des Fachgebiets oder Phrasen, die mit dem betreffenden Lemma zusammenhängen. Demuth entschied sich für informativere Artikel, die darüber hinaus noch etymologische Angaben, v. a. im Falle der Lemmata aus dem Französischen auch die Aussprache und manchmal auch grammatische Angaben anbieten.

Nach der Periodisierung von Alan Kirkness (1998:410-413) entstanden diese Nachschlagewerke in unterschiedlichen Phasen des Purismus. Die ersten zwei Auflagen von Sommers Werk stammen aus der Übergangsphase 1789-1819, in der das Thema des Purismus v. a. in den akademischen Kreisen heftig diskutiert wurde, während es in der breiten Öffentlichkeit keine wichtige Rolle spielte. Demuths Wörterbuch entstand dagegen in der Zeit des verstärkten Interesses an der Verdeutschungspraxis, das mit dem Streben nach der Einigung der Deutschen zusammenhing.

Es handelt sich um keine Verdeutschungswörterbücher im engeren Sinne, ${ }^{7} \mathrm{~d}$. h. Werke, in denen zu jedem Lemma lediglich Äquivalente (Verdeutschungen) aufgelistet werden, sondern die Wörterbuchartikel beinhalten sowohl Äquivalente als auch Paraphrasen. Aus diesem Grund gehören sie zu denjenigen Werken, die eine Kombination aus Verdeutschungs- und Fremdwörterbüchern darstellen ${ }^{8}$ (vgl. Sztandarska 2014:187 f., Janečková 2020:26 f.).

\subsection{Die Autoren der Wörterbücher}

Die Biographie von Johann Gottfried Sommer (geb. 1782 oder 1783 in Leuben bei Dresden, gest. am 11. oder 12.11.1848 in Prag) befindet sich in mehreren Nachschlagewerken aus dem 19. Jh. Es handelte sich anscheinend um eine bedeutende Persönlichkeit in der österreichischen Monarchie. In Dresden und später auch in Prag war Sommer Lehrer. In Prag war er als Professor für wissenschaftliche Gegenstände am Konservatorium tätig. Weiter redigierte er Zeitschriften und beteiligte sich u. a. an der Herausgabe des ,Taschenbuchs zur Verbreitung geographischer Kenntnisse', da er sich neben seiner Lehrtätigkeit mit Geographie beschäftigte. Für die Verfassung des Wörterbuchs spielte ohne Zweifel sein Studium der modernen Sprachen eine wichtige Rolle (für Weiteres vgl. Janečková 2020:19).

Die Bestimmung der Autorschaft erweist sich im Falle des zweiten Wörterbuchs als schwieriger. Der Autor wurde nicht nur mit dem Namen „Joseph“ bezeichnet, sondern in Börsenblättern bzw. in einer Werbung erschien auch die Variante „C. Demuth“ (URL 3). Das Werk wird manchmal dem Tafelschreiberdirektor Karel Josef Demuth (geb. 11.3.1807 in Tabor, gest. 9.2.1889 in Prag) zugeschrieben, der zwischen den Jahren 1844 und 1862 in Brünn lebte. Trotzdem scheint es unwahrscheinlich, dass diese Tatsache in den Lexikoneinträgen zu seiner Person nicht vorgekommen

Eine einfachere Struktur der Artikel ist laut Kirkness (1990:1173) eines der Merkmale von Verdeutschungswörterbüchern.

Viele dieser Werke wurden vom Allgemeinen Deutschen Sprachverein initiiert und veröffentlicht (vgl. Hauffen 1916/1917). Dazu zählen insbesondere einen speziellen Fachbereich betreffende Verdeutschungshefte (vgl. Sztandarska 2017).

8 Das Wort „Verdeutschungswörterbuch“ bedeutet in dieser Phase der Untersuchung v. a. ,Äquivalente enthaltend“ und nicht ,zu den Arbeiten der Sprachpuristen gezählt‘. Das gilt insbesondere für Demuths Wörterbuch, das noch nie analysiert wurde. 
wäre, zumal es sich um einen später in den Adelstand erhobenen Mann handelte, dessen Verdienste in seinen Biographien aufgezählt wurden (vgl. Otto 1905:286 f., AČP 1930:71 f.).

Dagegen kommt Josef Karel Demuth (geb. 1811, gest. 13.1.1867 in Brünn) für die Autorschaft des Wörterbuchs in Frage (URL 5). In seinem Leben wechselte er oft die Stelle. Er arbeitete u. a. als „Diurnist“”, „Privatbeamter“, „Cursor“ (Bote) eines Schutzvereins oder als „Oberlandesgerichtsoffizial“" (vgl. URL 6, URL 7, URL 8, URL 5). Eine wichtige Voraussetzung für die Verfassung eines Wörterbuchs hatte er dank seiner Stelle als „Collaborant am mährischen Wanderer“, einem Kalender (URL 9). In der Redaktion des Kalenders konnte er nützliche Kontakte herstellen, die später bei der Herausgabe des Wörterbuchs von Bedeutung sein konnten. ${ }^{9}$ Außerdem wurde der Kalender von Carl Winiker, dessen Verlag auch Demuths Wörterbuch veröffentlichte, mehrere Jahre lang verlegt. Weitere Beweise für die Verifizierung oder Ablehnung dieser Hypothese konnten leider nicht eruiert werden. Jedoch kann von der Voraussetzung ausgegangen werden, dass der Autor des zweiten Wörterbuchs im Gegensatz zu Sommer aus den böhmischen Ländern stammte. ${ }^{10}$

\section{Einstellungen zum Sprachpurismus}

Im Folgenden wird der Frage nachgegangen, ob sich die Autoren für den Sprachpurismus interessierten. Da in den Biographien keine näheren Informationen zur Beantwortung dieser Frage gefunden werden konnten, müssen Indizien dafür in den Metateilen ihrer Wörterbücher gesucht werden.

\subsection{Motive der lexikographischen Arbeit bei Sommer und Demuth}

Wie es später bei den radikaleren Mitgliedern des Allgemeinen Deutschen Sprachvereins der Fall war, spielten nationalistische und patriotische Motive auch bei Sommer eine Schlüsselrolle (vgl. Bernsmeier 1977). Aus dem Vorbericht (URL 12:III-XII) und der Vorrede (URL 12:XIV-XVII) zu seinem Wörterbuch geht ein Unbehagen an der damaligen politischen Situation hervor, d. h. an der Bedrohung des deutschsprachigen Gebiets durch Napoleons Truppen. Des Weiteren zitiert er eine patriotisch gefärbte Äußerung von Friedrich Ludwig Jahn, ${ }^{11}$ einem bekannten Sprachreiniger im Bereich der Sportlexik (für Weiteres vgl. Janečková 2020:19 f.).

Für Demuth und die Herausgeber seines Wörterbuchs scheint eine derartige Motivation nicht entscheidend zu sein. Zumindest hielten sie es nicht für wichtig, sie in der Vorrede (URL 12) anzuführen. Wie bereits im vorausgehenden Abschnitt angedeutet wurde, ging es ihnen v. a. um eine möglichst umfangreiche Lemmaliste, die Wörter aus verschiedenen Fachgebieten enthält. ${ }^{12}$ Das Wörterbuch zielte folglich kaum ausschließlich auf ein Publikum, das nichtindigene Ausdrücke vermeiden möchte, sondern eher auf diejenigen Wörterbuchbenutzer, die Texte, in denen solche Wörter vorkommen, besser verstehen wollten.

\subsection{Verdeutschungsprogramme}

Der Vorbericht zur ersten Auflage (URL 12:III-XII) und die Vorrede zur zweiten Auflage (URL 12:XIV-XVII) zeigen, dass sich Sommer für die Verdeutschungsarbeit interessierte und mit seinem Werk einen Beitrag dazu leisten wollte. Er war mit den puristischen Bestrebungen seiner Zeit

\footnotetext{
9 Der Herausgeber seines Wörterbuchs, Heinrich Pardatscher, war als Redakteur tätig (URL 10).

10 An dieser Stelle möchte ich mich bei Bc. Lenka Schindlerová für ihre große Hilfe bei der Suche nach Josef Karel Demuth bedanken. Des Weiteren will ich allen tschechischen Archiven sowie Fachleuten, die mir bei der Suche behilflich waren, meinen Dank aussprechen.

11 In diesem Zitat heißt es u. a.: „Klar wie des Teutschen Himmel, fest wie sein Land, ursprünglich wie seine Alpen, und stark wie seine Ströme, bleibe seine Sprache!“(URL 12:XII).

12 Nach den Herausgebern übertrifft Demuths Wörterbuch „die bedeutendsten Werke dieser Art fast um das Doppelte“ (URL 11).
} 
gut vertraut, insbesondere mit der Arbeit von Joachim Heinrich Campe. Sommer bemühte sich, die Reihen der Sprachpuristen zu erweitern, und legte sein kurzes Verdeutschungsprogramm vor, in dem er die Grundprinzipien seiner Verdeutschungsarbeit und die Bildung der idealen Verdeutschungen vorstellte. Diese sollten am besten einsilbig und wortbildungsfähig sein. Nicht geeignet ist nach seinem Verständnis deshalb die Verdeutschung in Form eines Kompositums, bei dem es sich lediglich um eine wörtliche Übersetzung des fremdsprachigen Ausdrucks handelt. Die von ihm empfohlenen Quellen zur Bildung neuer Verdeutschungen sind ältere Sprachstufen des Deutschen, Dialekte und andere germanische Sprachen. Man sollte keine gelehrten Ausdrücke und Modewörter in Verdeutschungswörterbücher aufnehmen, sondern nur bereits etablierte Wörter. Wie ein Widerspruch klingt seine Forderung nach sachlichen und kurzen Einträgen, die zugleich viele Erklärungen für ungelehrte und ungebildete Benutzer enthalten müssen. Er legte außerdem spezielle Anforderungen an Sprachreiniger fest. Idealerweise verdeutscht jeder Gelehrte nur die Wörter aus dem Fachgebiet, das er selbst versteht, z. B. er den Musikwortschatz. ${ }^{13}$

Die Vorrede des zweiten Wörterbuchs weist dagegen keinen Zusammenhang mit den puristischen Bestrebungen auf. Sie beschränkt sich auf die Hervorhebung der Anzahl der Lemmata und auf die Aufzählung der Fachgebiete, aus denen diese Lemmata stammen. Im Gegensatz zu Sommer sollten hier jedoch auch Fachwörter erscheinen. Der Autor dieses Werkes maß anscheinend, wie bereits angegeben, einer möglichst großen Lemmaliste eine Schlüsselrolle bei.

\section{Zwischenfazit}

Bereits der Blick auf die in den Abschnitten 2 und 3 behandelten Aspekte, die die beiden Autoren, ihre Einstellungen zum Purismus und die Wörterbuchstrukturen betreffen, zeigt recht unterschiedliche Umstände und Strategien, die Einfluss auf die lexikographische Arbeit beider Autoren hatten. Es gibt keine Belege dafür, dass Demuth bzw. die Herausgeber seines Wörterbuchs mit diesem Werk einen Beitrag zur puristischen Bewegung hätten leisten wollen. Vielmehr verdeutlicht das Wort „verdeutschend“, das sich im Titel des Wörterbuchs befindet, dass man im Wörterbuch nicht nur Paraphrasen, sondern auch Äquivalente der Lemmata findet. Die für den Sprachpurismus günstigere Entstehungszeit wirkte sich auf die Gestaltung des Wörterbuchs nicht aus.

Ein völlig anderes Bild ergibt sich aus den Informationen über Sommer. Er beabsichtigte offensichtlich, die puristischen Bestrebungen der damaligen Zeit durch sein Werk zu unterstützen. Aus dem Vorbericht und der Vorrede zu seinem Wörterbuch lassen sich ein kurzes Verdeutschungsprogramm und seine Motive der Verdeutschungsarbeit herauslesen. Das Grundwort im Kompositum „Verdeutschungswörterbuch“ erfüllt demnach die Aufgabe, auf den Zusammenhang mit den damaligen puristischen Bestrebungen hinzuweisen. ${ }^{14}$

Demuths Werk erfüllt deshalb anhand der theoretischen Informationen die Ziele eines Fremdwörterbuchs, während Sommers Werk mehr zu den puristisch orientierten Verdeutschungswörterbüchern tendiert. All diese Informationen sagen jedoch nichts darüber aus, ob bzw. wie diese Tatsachen die Gestaltung der Wörterbücher und die Lemmaliste beeinflussten.

\section{Analyse der Lemmata}

Am Beispiel der Lemmata, die zu den Wortstrecken E und L zählen, wird im Folgenden untersucht, ob die in den vorangehenden Abschnitten behandelten Tatsachen Einfluss auf die Gestaltung der Bedeutungsangaben hatten. Es werden besonders die Relation zwischen der Anzahl von Äquivalenten und Paraphrasen, ${ }^{15}$ die Form der Äquivalente, die Etymologie der Lemmata, und ggf. zusätzliche

13 Da Sommer die Verdeutschungen, die er in seinem Wörterbuch selbst bildete, nicht markierte, ist es fraglich, ob er diese Regel bei seiner Arbeit beachtete.

14 Für die theoretischen Überlegungen über die Funktion des Titels vgl. Janečková 2020.

15 Es wird davon ausgegangen, dass sich puristisch orientierte Lexikographen um eine möglichst große Anzahl von Verdeutschungen, die Wörter fremder Herkunft ersetzen sollen, bemühen. 
Informationen, die in den Wörterbuchartikeln vorkommen, berücksichtigt. Zum Schluss wird auf die Verwendung der Fraktur und der Antiquaschrift eingegangen.

Da sich die Lemmata in den einzelnen Wortstrecken bei Sommer und Demuth nicht vollkommen decken, wurden in die Analyse v. a. diejenigen Lemmata einbezogen, die in den beiden Werken zu finden sind. Die Unterschiede, die sich in der Lemmaauswahl finden lassen, können jedoch bei der Bewertung der spezifischen Strategien der Autoren und ihrer Einstellungen zum Sprachpurismus eine bedeutende Rolle spielen. Aus diesem Grund wird nach den Analyseergebnissen noch denjenigen Lemmata Aufmerksamkeit geschenkt, die nur in jeweils einem Wörterbuch erscheinen.

\subsection{Ergebnisse der Analyse}

Aus der Analyse der festgelegten Kriterien resultieren klare Ergebnisse. In der Wortstrecke E lassen sich 1053 Lemmata finden, die in den beiden Wörterbüchern vorkommen. Aus der Analyse ergibt sich trotz der Differenzen zwischen den Wörterbüchern, dass es keine größeren Unterschiede bei der Anzahl der Äquivalente und Paraphrasen bei Sommer und Demuth gibt. Die Bedeutungsangabe in Form eines Äquivalents wurde in beiden Werken bevorzugt, wie Tab. 1 veranschaulicht. Wie beim Buchstaben L erscheinen hier außerdem kombinierte Bedeutungsangaben, die sowohl aus einem Äquivalent als auch einer Paraphrase bestehen.

\begin{tabular}{|l|ll|ll|}
\hline E & \multicolumn{2}{|l|}{ Sommer } & Demuth \\
\hline Äquivalent & 674 & $64,01 \%$ & 687 & $65,24 \%$ \\
\cline { 1 - 2 } & 104 & $9,88 \%$ & 97 & $9,21 \%$ \\
\cline { 1 - 4 } beides & 275 & $26,12 \%$ & 269 & $25,55 \%$ \\
\hline
\end{tabular}

Tab. 1: Lemmata der Wortstrecke E

Die Analyse von 452 Lemmata mit dem Anfangsbuchstaben L bestätigt die Tendenz, die beim Buchstaben E festgestellt wurde. Auch hier überwiegen in den Bedeutungsangaben die Äquivalente (s. Tab. 2).

\begin{tabular}{|l|ll|ll|}
\hline $\mathbf{L}$ & \multicolumn{2}{|l|}{ Sommer } & \multicolumn{2}{|l|}{ Demuth } \\
\cline { 1 - 4 } Äquivalent & 236 & $52,21 \%$ & 249 & $55,09 \%$ \\
\cline { 1 - 5 } Paraphrase & 74 & $16,37 \%$ & 52 & $11,50 \%$ \\
\cline { 1 - 4 } beides & 142 & $31,42 \%$ & 151 & $33,41 \%$ \\
\hline
\end{tabular}

Tab. 2: Lemmata der Wortstrecke L

Auch im Fall der konkreten Verdeutschungsvorschläge sieht die Lage nicht anders aus. Das Angebot an Äquivalenten ist in beiden Wörterbüchern sehr ähnlich. Nur manchmal erscheint in einem Wörterbuch ein anderes Äquivalent, häufig deshalb, weil der Autor dieses Wörterbuchs mehrere Bedeutungen eines Lemmas anführt. Nur ausnahmsweise trifft man auf völlig unterschiedliche Möglichkeiten. Es wurden keine ungewöhnlichen Verdeutschungen gefunden, die bei Gegnern des Sprachpurismus eine heftige Kritik bzw. Spott hätten bewirken können. ${ }^{16}$ Auffällig sind lediglich einige Wortbildungen mit dem Suffix -ei, die heutzutage nicht mehr benutzt werden, z. B. Mondwandelei, Kundschafterei, Ausländerei, Briefstellerei oder Gesichtsschneiderei. Ausgewählte Beispiele der Äquivalente befinden sich in Tab. 3.

16 Wie z. B. Verdeutschungen von Gegenständen aus anderen Kulturen oder die Verwendung von umständlichen Komposita, die Campe betrieb (vgl. Polenz 1994:131, nach Daniels 1959). 


\begin{tabular}{|l|l|l|}
\hline Lemma & Äquivalente bei Sommer & Äquivalente bei Demuth \\
\hline Effarirt/effarirt & verstört, verblüfft & verstört, verblüfft \\
\hline Einrangiren/einrangiren & einreihen, einordnen, einstellen & einreihen, einstellen \\
\hline Longimetrie & Längenmessung, Längenmeßkunde & die Längenmessung ${ }^{17}$ \\
\hline Lithographie & Steinbeschreibung & $\begin{array}{l}\text { die Steinschreibung, die Steinzeichnung; } \\
\text { der Steindruck }\end{array}$ \\
\hline Libertin & Lüftling, Leichtfuß & ein Freigeist, Freidenker, Wüstling \\
\hline
\end{tabular}

Tab. 3: Lemmata und ihre Äquivalente

Bei beiden Autoren sind es v. a. Wörter aus dem Lateinischen, die hier als Lemmata erscheinen (bei E etwa 42\%, bei L etwa 52\%). Sommer widmete also dem Französischen keine größere Aufmerksamkeit, wie man aufgrund seines Wörterbuchvorspanns erwarten könnte (vgl. auch Janečková 2020). An den bis jetzt zusammengefassten Kriterien lässt sich die unterschiedliche Zielsetzung beider Publikationen nicht erkennen.

Da Demuths Wörterbuch eine viel umfangreichere Lemmaliste enthält, gibt es viele Ausdrücke, für die Sommer nur wenige Wörter aus einer Wortfamilie anführt, während Demuths Angebot breiter ist. So findet sich bei Sommer das Lemma Encephalognomik, aber nicht die Lemmata Encephalopathik, Encephalotomie. Manchmal ist es jedoch auch umgekehrt. Neben der Reduzierung der Lemmata aus derselben Wortfamilie schloss Sommer häufig sehr viele Ausdrücke aus, z. B. bietet Demuth zwischen Enallage und en blanc noch weitere 44 Lemmata, die bei Sommer nicht vorkommen. Außer nichtindigenen Wörtern dienen ab und zu auch einheimische Vokabeln als Lemmata. Besonders Demuth führt eine ganze Reihe von solchen Ausdrücken an, z. B. Eigenlöhner, Eisenglas, Eichelsteine, Eieröl, Eiermulm, sodass er manchmal auch den einheimischen bzw. längst eingebürgerten Wortschatz für erklärungsbedürftig hielt. Eine spezielle Kategorie der Lemmata stellen bei Demuth Personennamen, die nicht auf mythologische Gestalten verweisen (Eberhard, Edmund, Eginhard usw.), ${ }^{18}$ dar, während Sommer an den Anfang jeder Wortstrecke eine Liste mit Abkürzungen (wie $L$. S., Lb. oder etc.) stellt, die bei Demuth nicht zu finden ist.

Einen auffallenden Unterschied zwischen den Nachschlagewerken stellt die Verwendung der Fraktur- und der Antiquaschrift dar. Diese scheint darauf hinzuweisen, wie fremd die Lemmata nach Sommer und Demuth waren, d. h. ob es sich nach ihrer Einschätzung um im Deutschen bereits adaptierte nichtindigene Ausdrücke handelte. ${ }^{19}$ Bei Demuth wurden die meisten Lemmata in Frakturschrift gesetzt, während die Antiquaschrift selten vorkommt. Das beweisen auch die Analyseergebnisse, nach denen die Antiquaschrift nur bei weniger als 200 Lemmata verwendet wurde. Der Rest (mehr als 1300 Lemmata) ist in Fraktur. Die Antiquaschrift erscheint insbesondere dann, wenn es sich bei den Lemmata um Phrasen handelt (z. B. en faveur, ex animo und Lacrimae Christi), oder bei Ausdrücken, bei denen anscheinend der Grad an Integration sehr niedrig war, z. B. leges, licet und Esquire.

Sommers Wörterbuch zeichnet sich dagegen durch eine präzisere Unterscheidung der einzelnen Adaptationsgrade aus. In den analysierten Wortstrecken finden sich etwa 550 unadaptierte und mehr als 1000 adaptierte Lemmata. Die Benutzung der Antiquaschrift betrifft neben vielen Phrasen (wie Laesio enormis, Lettre de marque und Enfans perdus) auch zahlreiche Substantive (z. B. Eventail, Lever, Lictor) und weniger andere Wortarten (Largo, Ligato, Explicable). Es gibt viele Verben mit der Endung -iren, die als Ableitungen aus den nichtadaptierten Substantiven in Fraktur gesetzt

17 Im Gegensatz zu Sommer verwendet Demuth in den Bedeutungsangaben viel häufiger den bestimmten oder unbestimmten Artikel. In dieser Studie wird auf diesen Aspekt nicht näher eingegangen.

18 Dagegen erscheinen Namen aus der griechischen und römischen Mythologie sowohl bei Sommer als auch bei Demuth. Eine kurze Analyse der Personennamen in Verdeutschungswörterbüchern lässt sich in Sztandarskas Studie finden (vgl. Sztandarska 2016).

19 Die typographische Gestaltung von Werken konnten sowohl Schriftsteller als auch Verleger beeinflussen (vgl. Killius 1999:428-440). 
wurden, z. B. Expromittiren, Ebranliren, Lucriren. Der häufigere Einsatz der Antiquaschrift konnte ein wirksames Mittel der puristischen Bemühungen sein. Er hebt in gewissem Sinne die Notwendigkeit der Verdeutschungsarbeit hervor, indem das damalige Lesepublikum sah, wie viele nicht eingebürgerte Wörter es im Deutschen gab. ${ }^{20}$

\section{Schlussfolgerungen und Ausblick}

Im Rahmen dieser Studie wurde der Zusammenhang zweier Wörterbücher, die in Böhmen und Mähren entstanden, mit den sprachpuristischen Tendenzen im deutschsprachigen Raum hinterfragt, da beide Werke „Verdeutschung“ bzw. „,verdeutschend“ im Titel haben. Ein solcher Zusammenhang ist bei Johann Gottfried Sommer evident. Das bestätigt ein kurzes Verdeutschungsprogramm, das sich im Vorspann seines Wörterbuchs befindet, seine nationalistische Rhetorik und die Hervorhebung der Verdeutschungsarbeit anderer Gelehrter. Dagegen lässt sich schlussfolgern, dass im Falle von Joseph Demuth jegliche Beweise für sprachpuristische Motive seiner lexikographischen Arbeit fehlen. Er strebte anscheinend nach einem Werk, das den Benutzern möglichst viele Lemmata bieten sollte. In einem größeren Maß als Sommer berücksichtigte er in seinem Wörterbuch auch den einheimischen Wortschatz. Anhand dieser Beobachtungen, die sich v. a auf den ersten Teil der Studie stützen, kann Demuths Werk folglich nicht für die Arbeit eines Sprachpuristen gehalten werden.

Die Auswertung anderer Aspekte der untersuchten Nachschlagewerke erweist sich als schwieriger. Die analysierten Wörterbücher unterscheiden sich durch die Struktur der Wörterbuchartikel. Demuths informativeren Artikeln, die neben den Bedeutungsangaben und der Angabe des Fachgebiets auch Etymologie und ggf. die Aussprache der Lemmata und grammatische Angaben enthalten, steht Sommers einfachere Gestaltung der Artikel gegenüber, in denen er sich auf die Bedeutung, das betreffende Fachgebiet und manchmal feste Phrasen beschränkt. Da es jedoch auch andere Wörterbücher gibt, z. B. von Joachim Heinrich Campe oder Johann Christian August Heyse, die trotz zusätzlicher Angaben traditionell zu Verdeutschungswörterbüchern gezählt werden, lassen sich anhand dieses Kriteriums die Wörterbücher nicht voneinander abgrenzen (vgl. URL 13, URL 14). Bemerkenswert ist allerdings der Einsatz der Fraktur- und Antiquaschrift bei Lemmata. Durch die häufige Verwendung der Antiquaschrift in Sommers Wörterbuch wird die Fremdheit der betreffenden nichtindigenen Ausdrücke hervorgehoben. Demuth verwendete die Antiquaschrift lediglich für feste Phrasen und außerdem bei Lemmata, die wohl nach Demuth keine Integration aufwiesen. ${ }^{21}$ Eine genauere Untersuchung dieses Aspekts in der Fremdwortlexikographie und der Vergleich mit Sommers und Demuths Werken könnte interessante Ergebnisse liefern.

Was trotz aller angeführten Unterschiede keine Veränderung erfuhr, ist die Form der Bedeutungsangaben. Sowohl die Relation zwischen der Anzahl von Äquivalenten und Paraphrasen als auch die Form der Äquivalente zeigen keine maßgeblichen Differenzen. Anhand der Wörterbuchstruktur ist es folglich kaum möglich, Fremd- und eher puristisch orientierte Verdeutschungswörterbücher voneinander abzugrenzen. ${ }^{22}$ Deshalb spielen Verdeutschungsprogramme und andere Informationen theoretischer Art, u. a. die eigene Rhetorik des Autors oder seine Biographie, bei der Klassifizierung eine bedeutende Rolle.

Die sprachpuristisch ausgerichtete deutschsprachige Lexikographie in den böhmischen Ländern, insbesondere die vor der Gründung des Allgemeinen Deutschen Sprachvereins, bleibt jedoch weiterhin fast unerforscht. Angesichts der spezifischen bilingualen Sprachsituation in Böhmen und Mähren sollte die Suche nach weiteren Wörterbüchern, die dem von Sommer ähneln und von einem einheimischen Autor verfasst wurden, nicht aufgegeben werden.

20 Ein Vergleich mit anderen lexikographischen Werken könnte zur Entdeckung weiterer bedeutender Kriterien der Schriftverwendung führen. So erscheinen z. B. alle Lemmata bei Campe in der Antiquaschrift (vgl. URL 13).

21 Da jedoch Demuths Wörterbuch etwa 30 Jahre nach Sommers Werk erschien, muss gleichzeitig mit einer möglichen Veränderung des Status der betreffenden Lemmata gerechnet werden.

22 Falls die Wörterbücher keine nicht gelungenen oder anders „,verdächtigen“Verdeutschungsvorschläge beinhalten, wie es z. B. bei Campe und manchmal bei Zesen der Fall war (vgl. Polenz 1994:121, 131 f.). 


\section{Literaturverzeichnis}

\section{Primärliteratur:}

URL 1: Bote für Tirol und Vorarlberg. Nr. 168, 25. 7. 1850, S. 832. https://diglib.uibk.ac.at/obvuibz/ periodical/pageview/522470 [21.10.2020].

URL 2: Börsenblatt für den deutschen Buchhandel, 2. 3. 1852, S. 276. https://www.boersenblattdigital.de/pageview?tx_dlf\%5Bdouble $\% 5 \mathrm{D}=0 \& \mathrm{tx}$ delf\%5Bhighlight_word $\% 5 \mathrm{D}=$ Demuth\&tx dlf $\% 5 \mathrm{Bid} \% 5 \mathrm{D}=8695 \& \overline{\mathrm{tx}} \_\mathrm{dlf} \% 5 \mathrm{Bpage} \% 5 \mathrm{D}=4 \& \mathrm{cH}$ ash=ba4d91481a $\overline{8} 81 \mathrm{fce} 69009786 \mathrm{e} 3 \mathrm{cc} 94 \mathrm{a} \overline{5}$ [21.10.2020].

URL 3: Börsenblatt für den deutschen Buchhandel, 12.1.1855, S. 68. https://www.boersenblatt-digital. de/pageview?tx_dlf[highlight_word] $=$ Demuth\&tx_dlf[id] $=9534 \&$ tx_dlf[page] $=8 \& \mathrm{cHash}=9$ aa1e9c632355249df6e2e21359e-2bd3\&fbclid=IwAR3qTpY_xyf78Z7JZTVmKqE-QTN32-dWonzz4191r5onso8dEmJXI8F_DSo [21.10.2020].

URL 4: Oesterreichische Buchhändler-Correspondenz, Nr. 22, 1. 6. 1872, S. 208. http://anno.onb. ac.at $/$ cgi-content $/$ anno $?$ aid $=$ obc $\&$ datum $=18720601 \&$ seite $=8 \&$ zoom $=33 \&$ query $=\% 22$ Vollst $\% \mathrm{C} 3 \% \mathrm{~A} 4$ ndigstes $\% 2 \mathrm{Bverdeutschendes} \% 2 \mathrm{Bund} \% 2 \mathrm{Berk1} \% \mathrm{C} 3 \% \mathrm{~A} 4 \mathrm{rendes} \% 22 \& \mathrm{ref}=$ annosearch\&fbclid=IwAR2BxTrmjjTk0lbWU0h9gTdJp4CsWfiAuVEjraUC1Oxy6QTES4Xh5uti5Uc [21.10.2020].

URL 5: Moravský zemský archiv v Brně (17111). Sterbematrik Brno - Neposkvrněné početí P. Marie na Křenové (1867-1888). http://actapublica.eu/matriky/brno/prohlizec/8048/?strana=256\& fbclid=IwAR39iGrH7nt97eTYNQh2lhc7YyDxPppqKiJvFk6UOW23V2xN7PSwU6wZ0Wg [21.10.2020].

URL 6: Archiv města Brna, fond A 1/59 Archiv města Brna-Sčítání lidu z let 1850-1910, rkp. č. 2689, f. 99v, Protokol o sčítání lidu z roku 1850, Josef Demuth, Bäckergasse 5. http://digiarchiv.brno. cz/archive/id/dd822d20-f3c5-4bf6-be8f-a64f7724e 89b?datatext $=1850 * * * B \% C 3 \% A 4 c k e r g a s-$

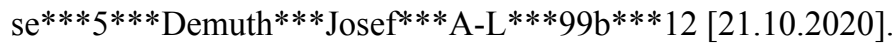

URL 7: Moravský zemský archiv v Brně (17156). Geburtsmatrik Brno - Staré Brno u Nanebevzetí Panny Marie (1848-1862), S. 36. http://actapublica.eu/matriky/brno/prohlizec/8084/?strana=19 [21.10.2020].

URL 8: Moravský zemský archiv v Brně (17155). Geburtsmatrik Brno - Staré Brno u Nanebevzetí Panny Marie (1832-1848), S. 509. http://actapublica.eu/matriky/brno/prohlizec/8083/?strana $=262$ [21.10.2020].

URL 9: Moravský zemský archiv v Brně (17162). Trauungsbuch Brno - Staré Brno u Nanebevzetí Panny Marie (1784-1947), S. 305. http://actapublica.eu/matriky/brno/prohlizec/8088/?stra$\mathrm{na}=154[21.10 .2020]$.

URL 10: Archiv města Brna, fond A 1/59 Archiv města Brna - Sčítání lidu z let 1850-1910, rkp. č. 2705, f. 9r, Protokol o sčítání lidu z roku 1870, Heinrich Pardatscher, Rudolfgasse 25. http:// digiarchiv.brno.cz/archive/id/74d8c4a1-9a1c-4fd0-843e-4f817c75a8b8?datatext=1870***Rudo lfsgasse ${ }^{* * * 25 * * * \text { Pardatscher***} \text { Heinrich }^{* * *} \mathrm{P}-\mathrm{R} * * * 9 \mathrm{a} * * * 7}$ [21.10.2020].

ULR 11: Demuth, Joseph (1853): Vollständigstes verdeutschendes und erklärendes FremdwörterBuch der deutschen Schrift= und Umgangssprache, mit Bezeichnung der richtigen Aussprache, Betonung, Abstammung, Rechtschreibung und Bildung der Wörter, so wie erklärendes Wörterbuch aller in der deutschen Sprache gebräuchlichen Wörter der Asthetik, Musik, Technik, Schifffahrtskunde, Botanik, Naturwissenschaft, Kriegswissenschaft, Eigennamen etc. Brünn. http://www. digitalniknihovna.cz/mzk/view/uuid:216cfbe0-cf50-11e2-8f90-005056827e51 ?page=uuid:a316c0c0-f0f4-11e2-a0b3-5ef3fc9bb22f [21.10.2020].

ULR 12: Sommer, Johann Gottfried (1819): Neuestes wort- und sacherklärendes Verteutschungswörterbuch aller jener aus fremden Sprachen entlehnten Wörter, Ausdrücke und Redensarten, welche die Teutschen bis jetzt, in Schriften und Büchern sowohl als in der Umgangssprache, noch immer für unentbehrlich und unersetzlich gehalten haben; verbunden mit einer Erklärung auch der weniger bekannten Kunstwörter und andern Ausdrücke der teutschen Sprache. Ein höchstnützliches Handbuch für Geschäftsmänner, Zeitungsleser, und alle gebildete Menschen 
überhaupt. Prag. https://books.google.cz/books?id=Sn9cAAAAcAAJ\&printsec=frontcover\&hl $=\mathrm{cs} \# \mathrm{v}=$ onepage \&q\&f $\mathrm{f}=$ false $[21.10 .2020]$.

ULR 13: CAMPE, Joachim Heinrich (1813): Wörterbuch zur Erklärung und Verdeutschung der unserer Sprache aufgedrungenen fremden Ausdrücke: ein Ergänzungsband zu Adelung's und Campe's Wörterbüchern. Braunschweig. https://books.google.cz/books?id=xJNEAAAAcAAJ$\&$ printsec $=$ frontcover $\& \mathrm{hl}=\mathrm{cs} \# \mathrm{v}=$ onepag $\& \mathrm{q} \& \mathrm{f}=$ false $[21.10 .2020]$.

ULR 14: Heyse, Johann Christian August (1840): Kleines Fremdwörterbuch zur Verdeutschung und Erklärung aller in unserer Schrift- und Umgangssprache üblichen fremden Ausdrücke, mit Bezeichnung der Aussprache und Betonung, und Andeutung ihrer Herkunft : Ein reichhaltiger Auszug aus dem allgemeinen Fremdwörterbuche. Hannover. https://books.google.cz/ books? $\mathrm{id}=$ Cf5IAAAAcAAJ\&printsec $=$ frontcover $\& \mathrm{hl}=\mathrm{cs} \# \mathrm{v}=$ onepage $\& \mathrm{q} \& \mathrm{f}=$ false $[21.10 .2020]$.

\section{Sekundärliteratur:}

AČP: Almanach československých právníků. Životopisný slovník čs. právníků, kteři působili $v$ umění, vědě, krásném písemnictví a politice od Karla IV. počinaje až na naše doby. K III. sjezdu čs. právníků v Bratislavě s podporou české akademie a ministerstva spravedlnosti vydal JUDr. Michal Navrátil. Praha 1930.

BernsmeIER, Helmut (1977): Der Allgemeine Deutsche Sprachverein in seiner Gründungsphase. In: Muttersprache 87, S. 369-395.

DANIELs, Karlheinz (1959): Erfolg und Mißerfolg der Fremdwortverdeutschung. Schicksal der Verdeutschungen von J. H. Campe. In: Muttersprache 69, S. 46-54, 105-114, 141-146, zitiert bei Polenz 1994, S. 131.

Hauffen, Adolf (1916/1917): Der Allgemeine Deutsche Sprachverein und Deutschböhmen I, II. In: Deutsche Arbeit 16, H. 7/8 und 9, S. 296-302, 345-351.

Killius, Christina (1999): Die Antiqua-Fraktur Debatte um 1800 und ihre historische Herleitung. Wiesbaden.

Kirkness, Alan (1990): Das Fremdwörterbuch. In: Hausmann, Franz Josef / Reichmann, Oskar / Wiegand, Herbert Ernst / ZGusta, Ladislav (Hrsg.): Wörterbuch. Ein internationales Handbuch zur Lexikographie. 2. Teilband. Berlin; New York, S. 1 168-1 178.

KiRKness, Alan (1998): Das Phänomen des Purismus in der Geschichte des Deutschen. In: Besch, Werner / Betten, Anne / Reichmann, Oskar / Sonderegger, Stefan (Hrsg.): Sprachgeschichte: ein Handbuch zur Geschichte der deutschen Sprache und ihrer Erforschung. 1. Teilband. Berlin; New York, S. 407-416.

JANEČKOVÁ, Eva (2020): Sprachpuristische Bestrebungen des 19. Jahrhunderts am Beispiel des Verdeutschungswörterbuchs von Johann Gottfried Sommer. In: Brünner Beiträge zur Germanistik und Nordistik 34 (1), S. 17-29.

Otto: Ottưv slovník naučný. Bd. 7: Dánsko - Dřevec. Praha 1893.

Polenz, Peter von (1994): Deutsche Sprachgeschichte vom Spätmittelalter bis zur Gegenwart. Band II: 17. und 18. Jahrhundert. Berlin; New York.

Schiewe, Jürgen (1988): Sprachpurismus und Emanzipation. Joachim Heinrich Campes Verdeutschungsprogramm als Voraussetzung für Gesellschaftsveränderungen. Hildesheim; Zürich; New York.

SzTANDARSKA, Katarzyna (2014): Zu Motiven der Verdeutschung von Fremdwörtern in den Spezialverdeutschungswörterbüchern. In: Annales Neophilologiarum 8, S. 187-202.

SzTANDARSKA, Katarzyna (2016): Vornamen in Wörterbüchern mit puristischen Zielen. In: Studia Niemcoznawcze LVIII, S. 613-624.

SzTANDARSKa, Katarzyna (2017): Verdeutschungshefte im Deutschen als Werkzeug im Kampf gegen Wörter fremder Herkunft. In: Studia Niemcoznawcze LX, S. 835-846.

\section{Internetquellen:}

ULR 15: Duden. http://www.duden.de/ [21.10.2020].

ULR 16: Digitales Wörterbuch der deutschen Sprache. https://www.dwds.de/ [21.10.2020]. 